tamment (parfois même dans la proportion de $50 \%$ sur la microflore totale) différents types de mamocoques (parmi lesquels il faut compter aussi Streptococcus liquefaciens) dans le lait des cuvettes (Stotzenmilch) pour la fabrication de l'Emmental, conformément à ce qui se vérifie dans le lait des cuves de maturation pour la fabrication du fromage Parmesan. On doit en outre considérer que les protéases des mamocoques poursuivent leur activité même après la mort des cellules.

Et encore : DoRnER même n'a-t-il pas observê, avec EREKson, que parmi huit Emmental le mieux réussi comme structure et comme goût a été celui dont le lait en chaudière contenait le plus grand nombre de coccus acidoprotéolytiques (Journal Bact., 29, $70,1935)$.

\title{
REVUE
}

\section{LE TRAITEIMENT DES EAUX RÉSIDUAIRES DE LAITERIE}

\author{
par \\ G. GÉNIN. \\ Ingénieur chimiste E. P. C. I.
}

La question de l'épuration des eaux résiduaires fournies par les diverses branches des industries chimiques et alimentaires est une de celles qui est particulièrement suivie par les administrations chargées de combattre l'empoisonnement des rivières.

Une très importante conférence sur ce sujet a eu lieu récemment en Angleterre, au cours de laquelle a été présentée une communication du $\mathrm{D}^{\mathrm{r}} \mathrm{A}$. PARker sur la question du traitement et de l'évacuation des eaux résiduaires.

En ce qui concerne plus particulièrement ce pays, e'est tout d'abord une Commission royale qui fut chargée de 1898 à 1915 d'entreprendre une vaste enquête sur les méthodes de traitement des eaux résiduaires industrielles et de procéder à des essais de laboratoire et industriels sur l'efficacité des méthodes dont on disposait à l'époque pour purifier les eaux industrielles. Les résultats de l'enquête entreprise à l'époque furent publiés dans 9 rapports consécutifs dont la conclusion essentielle était que ces eaux résiduaires pouvaient être classés en deux groupes :

a) Celles pour lesquelles une épuration pouvait être considérée comme réalisable.

b) Celles pour lesquelles l'épuration était irréalisable, tout au moins avec les procédés dont on disposait à l'époque. 
A la suite de cette première enquête, aucun décret ne fut pris concernant les obligations des industriels et la question fut seulement reprise en 1927 par la création d'un "Water Pollution Research Board " chargé par le "Department of Scientific and Industrial Research " de soumettre des plans d'étude pour là recherche de procédés permettant l'épuration des eaux résiduaires.

Depuis 1927, cette Commission a procédé à un très grand nombre d'essais de laboratoire et semi-industriels en vue de déterminer l'efficacité des différents procédés qu'on lui a signalés comme susceptibles d'être employés pour l'épuration des eaux résiduaires fournies par diverses industries. Mais cette Commission a tout d'abord constaté avant tout qu'il importait de remonter à l'origine de ces eaux résiduaires et de demander aux industriels, par une modification de leurs procédés et méthodes de fabrication, de réduire et même de supprimer complètement la production de ces eaux résiduaires. On a même pu montrer dans certains cas qu'en prenant cette précaution, on créait pour l'industriel consciencieux une source de profits souvent ignorée.

Néanmoins, il existe des cas où la production d'eaux résiduaires semble inévitable et la Commission a donc examiné le mode de traitement de ces eaux. Ce sont ces procédés qui ont été passés en revue par le Dr A. Parker.

Au cours de ces récentes années, l'importance de la question du traitement des eaux résiduaires de laiterie ou d'autres industries dans lesquelles on emploie le lait comme matière première a grandi avec le développement de cette industrie et la création d'un grand nombre de dépôts et de petites usines qui reçoivent, en vue de leur transformation, ou simplement de leur expédition, les laits fournis par les fermes des alentours. La multiplication de ces petites installations, qui rejetaient dans les rivières les plus voisines leurs eaux résiduaires, a entraîné pour le voisinage de très importants inconvénients, par suite d'une pollution parfois grave des rivières.

Depuis 4 ans environ le "Water Pollution Research Board" a donc poussé activement ses travaux dans ce domaine et une première étude a permis de montrer que, même dans les seuls dépôts qui se contentent d'assurer la collecte et la réexpédition du lait, après avoir néanmoins épuré et pasteurisé ee lait, les eaux de nettoyage de l'installation : nettoyage des réfrigérants, des pasteuriseurs, des réservoirs et du sol, entraînent ávec elles environ 0,5 à $1 \%$ du lait traité. Ceci signifie donc que dans un dépôt expédiant chaque jour 40 à 50.000 litres de lait, il y a environ 200 à 500 litres de lait perdus dans les eaux de lavage.

En ce qui concerne la relation qui existe entre le volume total du lait traité et la quantité d'eau rejetée à l'égout, on a constaté 
que cette relation varie dans de grandes proportions suivant les différents dépôts. Si on admet, en première approximation, que le volume d'eau de lavage est à peu près le même que le volume de lait traité, on voit qu'un dépôt traitant par jour 50.000 litres de lait doit rejeter à l'égout 50.000 litres d'eau contenant 0,5 à $1 \%$ de lait.

Or, des essais ont montré qu'en supposant que l'eau puisse être aérée dans des bassins pendant une durée de 5 jours, l'eau contenant $1 \%$ de lait absorbe 120 parties d'oxygène pour 100.000 parties d'eau, et le calcul montre alors qu'une installation qui rejette chaque jour 50.000 litres d'eau résiduaire contenant $1 \%$ de lait pollue la rivière absolument comme le feraient les eaux domestiques rejetées par une localité d'environ 1.200 habitants.

Si maintenant on envisage le cas de fabriques de lait concentré, de fromages, de beurre, ou d'autres produits laitiers, aux eaux de lavage du matériel vont s'ajouter d'autres produits résiduaires, comme par exemple les eaux provenant du lavage des évaporateurs, des bacs à fromage, des barattes, etc. Même si on exerce le plus grand soin pour éviter des pertes inutiles de lait qui constituent la matière principale de la fabrication ou de ses sous-produits comme le lait écrémé, le petit-lait, le sérum, ete., on peut admettre en première approximation que les eaux résiduaires provenant de ces installations contiennent une quantité de matières organiques qui correspond à celle que l'on trouverait dans ces eaux si on leur ajoutait environ $2 \%$ du lait utilisé. On a même vu fréquemment dans les fabriques de fromages ou de beurre que la quantité de matières organiques contenues dans les eaux résiduaires correspondait à 3 ou $4 \%$ du lait traité et même dans certains cas exceptionnels à 6 à $8 \%$.

On cite même en Angleterre le cas d'usines qui, il y a de nombreuses années, considéraient le petit-lait et le sérum comme de simples liquides résiduaires et avaient rejeté ces produits dans les rivières les plus proches, avec des conséquences désastreuses. Pour ne citer qu'un seul exemple, il faut pour oxyder les matières organiques contenues dans le sérum 4 à 5.000 parties d'oxygène pour 100.000 parties de liquide et ce produit est donc au moins 100 fois plus dangereux que les simples eaux d'égouts provenant d'une agglomération. Heureusement, on a ajourd'hui reconnu que le sérum et le petit-lait doivent être considérés comme des sousproduits de valeur et non comme des déchets; ils possèdent une valeur alimentaire indiscutable et peuvent être utilisés comme aliments ou pour la préparation des aliments. D'ailleurs, il existe aujourd'hui un marché du petit-lait et du sérum desséchés, et ce marché ne fait que croître.

Après une étude approfondie de la question, le « Water Pollu- 
tion Research Board " a été amené à la conclusion que l'on pourrait diminuer dans des proportions considérables la quantité de matières organiques dangereuses qui sont rejetées dans les eaux d'égout des laiteries, en apportant aux installations existantes des modifications simples et peu coûteuses, en modifiant également peut-être légèrement les procédés de fabrication et en apportant plus de soin aux opérations de nettoyage dans l'usine.

On a par exemple constaté qu'en disposant un bassin de décantation au-dessous de chacun des principaux appareils de l'installation et en laissant égoutter ces appareils dans ces bassins, avant de, procéder à leur nettoyage, on peut déjà réduire de moitié la quantité de matières organiques rejetées par les eaux de lavage. Or, l'économie réalisée en recueillant le liquide qui s'égoutte des appareils peut être considérable. Pour un dépôt qui traite par exemple 50.000 litres de lait par jour, cette économie annuelle peut correspondre à la valeur de 50.000 litres de lait et un simple calcul permet alors de constater que les frais de création de ces bacs d'égouttage pourraient être rapidement amortis par l'économie réalisée sur le lait. Cette économie pourrait même servir à amortir rapidement les installations destinées à l'épuration des eaux résiduaires.

En ce qui concerne le lavage des appareils servant à la fabrication du fromage, du beurre, etc., il y a même intérêt à pousser plus loin les précautions à prendre et on peut par exemple, après un bon égouttage, laver ces appareils avec une très faible quantité d'eau et ajouter ces eaux au liquide d'égouttage. Ce n'est qu'à ce moment alors qu'on procède à un lavage à grande eau.

On voit done que, sans entrer dans le vif du sujet, la Commission anglaise a déjà établi que l'on pourrait diminuer considérablement les risques d'empoisonnement des rivières par plus de précaution dans les opérations de l'usine et il a même été ealculé que, pour toute l'Angleterre qui pourtant n'est pas un important pays producteur de lait, l'économie annuelle réalisée, en prenant ces précautions, pourrait se chiffrer par 150.000 livres, soit au cours actuel environ 22 millions de franes.

\section{BIBLIOGRAPHIE ANALYTIQUE}

\section{JOURNAUX, REVUES, SOGIÉTÉS SAVANTES PHYSIOLOGIE}

Asimov (G. J.) et Krouze (N. K.). - Les préparations lactogéniques de la Pituitaire antérieure et l'augmentation 